MATHEMATICS OF COMPUTATION

Volume 69, Number 231, Pages 911-928

S 0025-5718(99)01165-5

Article electronically published on August 20, 1999

\title{
LOCKING-FREE FINITE ELEMENTS FOR THE REISSNER-MINDLIN PLATE
}

\author{
RICHARD S. FALK AND TONG TU
}

\begin{abstract}
Two new families of Reissner-Mindlin triangular finite elements are analyzed. One family, generalizing an element proposed by Zienkiewicz and Lefebvre, approximates (for $k \geq 1$ ) the transverse displacement by continuous piecewise polynomials of degree $k+1$, the rotation by continuous piecewise polynomials of degree $k+1$ plus bubble functions of degree $k+3$, and projects the shear stress into the space of discontinuous piecewise polynomials of degree $k$. The second family is similar to the first, but uses degree $k$ rather than degree $k+1$ continuous piecewise polynomials to approximate the rotation. We prove that for $2 \leq s \leq k+1$, the $L^{2}$ errors in the derivatives of the transverse displacement are bounded by $C h^{s}$ and the $L^{2}$ errors in the rotation and its derivatives are bounded by $C h^{s} \min \left(1, h t^{-1}\right)$ and $C h^{s-1} \min \left(1, h t^{-1}\right)$, respectively, for the first family, and by $C h^{s}$ and $C h^{s-1}$, respectively, for the second family (with $C$ independent of the mesh size $h$ and plate thickness $t$ ). These estimates are of optimal order for the second family, and so it is locking-free. For the first family, while the estimates for the derivatives of the transverse displacement are of optimal order, there is a deterioration of order $h$ in the approximation of the rotation and its derivatives for $t$ small, demonstrating locking of order $h^{-1}$. Numerical experiments using the lowest order elements of each family are presented to show their performance and the sharpness of the estimates. Additional experiments show the negative effects of eliminating the projection of the shear stress.
\end{abstract}

\section{INTRODUCTION}

In this paper we analyze two families of triangular finite elements for the approximation of the Reissner-Mindlin plate equations, paying particular attention to the issue of "locking," a problem which causes poor approximation for thin plates, and which typically occurs when standard low-order finite elements are used.

The Reissner-Mindlin model describes the deformation of a plate subject to a transverse loading in terms of the transverse displacement $\omega$ of the midplane and the rotation $\phi$ of fibers normal to the midplane. More precisely, $\omega$ and $\phi$, defined on the middle surface $\Omega$ of the plate, are determined as the solution of the partial differential equations

$$
\begin{gathered}
-\operatorname{div} \mathcal{C} \mathcal{E}(\phi)-\lambda t^{-2}(\operatorname{grad} \omega-\phi)=0 \quad \text { in } \Omega, \\
-\lambda t^{-2} \operatorname{div}(\operatorname{grad} \omega-\phi)=g \quad \text { in } \Omega
\end{gathered}
$$

Received by the editor August 14, 1998.

1991 Mathematics Subject Classification. Primary 65N30, 73K10, 73K25.

Key words and phrases. Reissner-Mindlin plate, finite element, locking-free.

The first author was supported by NSF grant DMS-9704556. 
subject to appropriate boundary conditions. Here $t$ is the plate thickness, $\mathcal{E} \boldsymbol{\phi}$ is the symmetric part of the gradient of $\phi, g$ is the scaled transverse loading function, and $\lambda=E k /[2(1+\nu)]$, where $E$ is Young's modulus, $\nu$ is Poisson's ratio, and $k$ the shear correction factor. For all $2 \times 2$ symmetric matrices $\boldsymbol{\tau}, \mathcal{C} \boldsymbol{\tau}$ is defined by

$$
\mathcal{C} \boldsymbol{\tau}=\frac{E}{12\left(1-\nu^{2}\right)}[(1-\nu) \boldsymbol{\tau}+\nu \operatorname{tr}(\boldsymbol{\tau}) I] .
$$

In this paper we shall consider the case of the hard clamped plate, which corresponds to the boundary conditions

$$
\omega=0, \quad \phi=0 \quad \text { on } \partial \Omega .
$$

Introducing the shear stress $\gamma=\lambda t^{-2}(\operatorname{grad} \omega-\phi)$, a weak formulation of this problem follows.

Find $\phi \in \stackrel{\circ}{H}^{1}(\Omega), \omega \in \stackrel{\circ}{H}^{1}(\Omega)$, and $\gamma \in \boldsymbol{L}^{2}(\Omega)$ such that

$$
\begin{gathered}
a(\boldsymbol{\phi}, \boldsymbol{\psi})+(\gamma, \operatorname{grad} v-\boldsymbol{\psi})=(g, v) \text { for all } \boldsymbol{\psi} \in \stackrel{\circ}{\boldsymbol{H}}^{1}(\Omega), v \in \stackrel{\circ}{H}^{1}(\Omega), \\
(\operatorname{grad} \omega-\boldsymbol{\phi}, \boldsymbol{\eta})-\lambda^{-1} t^{2}(\boldsymbol{\gamma}, \boldsymbol{\eta})=0, \quad \text { for all } \boldsymbol{\eta} \in \boldsymbol{L}^{2}(\Omega),
\end{gathered}
$$

where $a(\phi, \psi)=(\mathcal{C E}(\phi), \mathcal{E}(\boldsymbol{\psi}))$ and $(\cdot, \cdot)$ denotes the $L^{2}$ inner product in $\Omega$.

Many of the finite element methods which have been proposed to overcome the problem of "locking" have the following variational formulation. Find $\phi_{h} \in \boldsymbol{\Theta}_{h}$, $\omega_{h} \in W_{h}, \gamma_{h} \in \Gamma_{h}$ such that

$$
\begin{gathered}
a\left(\boldsymbol{\phi}_{h}, \boldsymbol{\psi}\right)+\left(\gamma_{h}, \operatorname{grad} v-\boldsymbol{R}_{h} \boldsymbol{\psi}\right)=(g, v) \quad \text { for all } \boldsymbol{\psi} \in \boldsymbol{\Theta}_{h}, v \in W_{h}, \\
\left(\operatorname{grad} \omega_{h}-\boldsymbol{R}_{h} \boldsymbol{\phi}_{h}, \boldsymbol{\eta}\right)-\lambda^{-1} t^{2}\left(\boldsymbol{\gamma}_{h}, \boldsymbol{\eta}\right)=0 \quad \text { for all } \boldsymbol{\eta} \in \boldsymbol{\Gamma}_{h},
\end{gathered}
$$

where $\boldsymbol{\Theta}_{h}, W_{h}$, and $\boldsymbol{\Gamma}_{h}$ are finite-dimensional subspaces of $\stackrel{\circ}{\boldsymbol{H}}^{1}(\Omega), \stackrel{\circ}{H}^{1}(\Omega)$, and $\boldsymbol{L}^{2}(\Omega)$, respectively, and $\boldsymbol{R}_{h}$ is an interpolation or projection operator defined on an appropriate space and mapping into $\boldsymbol{\Gamma}_{h}$. In some cases, the spaces are nonconforming and the differential operators in the formulation are applied on each element. The elements we consider in this paper are conforming elements which fit the above framework.

The lowest order element of the first family we consider is an element proposed by Zienkiewicz and Lefebvre 23. It approximates $\omega$ by continuous piecewise quadratics, $\phi$ by continuous piecewise quadratics plus quartic bubble functions (i.e., elements which are polynomials of degree $\leq 4$ on each triangle of the triangulation and vanish on all the triangle boundaries), and $\gamma$ by discontinuous piecewise linear functions. The operator $\boldsymbol{R}_{h}$ is an $\boldsymbol{L}^{2}$ projection. For general $k \geq 1$, this family uses continuous $(k+1)$-degree piecewise polynomials to approximate the transverse displacement, continuous $(k+1)$-degree piecewise polynomials enriched by $(k+3)$-degree bubble functions for the rotation, and $k$-degree discontinuous piecewise polynomials to approximate the shear stress. We prove that for $2 \leq s \leq k+1$, the approximate values of the derivatives of the rotation converge with order $h^{s-1} \min \left(1, h t^{-1}\right)$ in $L^{2}$, the values of the rotation converge with order $h^{s} \min \left(1, h t^{-1}\right)$ in $L^{2}$, and the derivatives of the transverse displacement converge with order $h^{s}$ in $L^{2}$. In both cases the constant in the estimate is independent of the mesh parameter $h$ and the plate thickness $t$. Note that the estimate for the derivative of the transverse displacement is of optimal order, while there is a deterioration of order $h$ in the approximation of both the rotation and its derivatives for small $t$, demonstrating 
locking of order $h^{-1}$. There is no improvement in the order of convergence for the approximation of $\omega$ itself for small $t$, as shown by computations reported in this paper.

The second family we consider is similar to the first, except that the rotation is approximated by continuous $k$-degree piecewise polynomials enriched by $(k+3)$ degree bubble functions. We prove that for $2 \leq s \leq k+1$, the approximate values of the derivatives of the rotation converge with order $h^{s-1}$ in $L^{2}$, and the values of the rotation and the derivatives of the transverse displacement converge with order $h^{s}$ in $L^{2}$. In this case, the order of convergence for the approximation of the derivative of the transverse displacement, the rotation, and the derivatives of the rotation are all optimal order, so this family of methods is free of locking. Again, computations show there is no improvement in the order of convergence for the approximation of $\omega$ itself.

In this paper we provide some numerical tests of the lowest order elements in both these families which confirm the order of convergence results discussed above. Further numerical experiments show the negative effects of eliminating the projection operator $\boldsymbol{R}_{h}$. Additional numerical results can be found in $\mathrm{Tu}[22$.

The second family described above appears to be a new family of locking-free elements for the Reissner-Mindlin plate. A rigorous error analysis of the method proposed in 23] and its generalization is also new.

There has been considerable progress in recent years on designing new lockingfree elements and in proving that elements previously proposed in the literature are in fact free of locking. We mention several which fit the framework discussed above. Combining ideas from the design of stable mixed finite elements for the Stokes problem and second order elliptic problems, Bathe, Brezzi, and Fortin [7] proposed several families of elements and proved error estimates for the limiting case $t=0$. The simplest triangular element in their families approximates the transverse displacement by continuous piecewise quadratics, the rotation by continuous piecewise quadratics plus cubic bubble functions, and interpolates the shear stress in the second lowest order rotated Raviart-Thomas space. For $t>0$, Brezzi, Fortin, and Stenberg [10] (see also Peisker and Braess [17]) completed the error analysis for the family of finite elements given in [7], deriving error estimates uniformly valid with respect to the thickness for all variables. Experimental results for some of these elements can be found in Bathe, Brezzi, and Cho 6]. A similar low order element was analyzed by Durán and Liberman [12]. It uses continuous, piecewise linear elements to approximate $\omega$ and continuous, piecewise linear elements plus the span of $\lambda_{2} \lambda_{3} \tau_{1}, \lambda_{3} \lambda_{1} \tau_{2}$, and $\lambda_{1} \lambda_{2} \tau_{3}$ as the trial space for the rotation, where $\left\{\lambda_{i}\right\}_{1 \leq i \leq 3}$ are the barycentric coordinates and $\tau_{i}$ is the tangential vector to the edge $i$ of the element $T$. To avoid locking, the shear stress is interpolated into the lowest order rotated Raviart-Thomas space. This element is shown to be free of locking and optimal in order. Durán and Liberman [13] also analyzed the convergence of a related element proposed by Zienkiewicz, Taylor, Papadopoulos, and Oñate 24], in which $\omega$ is now approximated by continuous, piecewise linear elements plus the space spanned by $\lambda_{2}^{2} \lambda_{3}, \lambda_{2} \lambda_{3}^{2}, \lambda_{1}^{2} \lambda_{3}, \lambda_{1} \lambda_{3}^{2}, \lambda_{1}^{2} \lambda_{2}$, and $\lambda_{1} \lambda_{2}^{2}$. This element is also shown to be of optimal order and free of locking.

Arnold and Falk 2] proposed a simple finite element which uses nonconforming linear finite elements to approximate the transverse displacement, conforming linear finite elements augmented by cubic bubbles to approximate the rotation, and projects the shear stress into the space of piecewise constant vectors. They show 
that the method has an optimal order of convergence independent of the plate thickness. The cubic bubble functions can be eliminated by static condensation, producing a method only involving linear elements but with a slightly perturbed stiffness matrix (cf. Arnold [1]). A generalization of this method with a somewhat simpler stiffness matrix has been developed independently by Durán, Ghioldi, and Wolanski [11, and Franca and Stenberg [14. Some results of numerical computations with these methods are reported in Franca Stenberg, and Vihinen [20, and Stenberg and Vihinen [21.

Nonconforming elements are also used in the scheme proposed by Oñate, Zarate and Flores [16. This method uses nonconforming linear elements as the trial space for the rotations and conforming linear elements as the trial space for the transverse displacement. The shear stress is interpolated into a rotated space of lowest order Raviart-Thomas elements. Arnold and Falk 5] analyzed this element and proved that the method gives optimal order error estimates uniform in $t$ when $t \leq h$, but that the method does not converge as $h$ goes to zero for $t$ fixed.

A unified approach for error analysis, which can be applied to many of the elements described above, can be found in R. Durán and E. Liberman 12. Another general approach to error analysis can be found in Pitkäranta and Suri 19]. The degrees of freedom for some of the elements described above are summarized in Figure 1.

There are several other approaches for finite element approximation schemes which are not based on the modified variational formulation (1.3)-(1.4), but which also produce error estimates uniform in the plate thickness. In important early work by Brezzi and Fortin [9, the Helmholtz decomposition was used to obtain a new variational formulation of the Reissner-Mindlin model, using $\omega$ and $\phi$ and two additional unknown functions. The drawback to this approach is that because of the additional unknowns, the resulting discrete problem is more complicated to solve.

Hughes and Franca [15] proposed a "stabilized" finite element scheme by modifying the Galerkin variational formulation to include least-square residual forms of the moment equilibrium equation and transverse shear constitutive equation. They proved that as long as sufficiently high-order finite elements are used, the method converges uniformly for all values of the plate thickness.

There are also methods which view the Reissner-Mindlin model as a penalized form of the Kirchoff plate model and are therefore appropriate in the limiting case $t=0$. An example of such a method along with a mathematical analysis can be found in Pitkäranta 18 .

Branble and Sun [8] introduced a least squares discretization based on the Helmholtz decomposition reformulation of Brezzi and Fortin to approximate the solution of the Reissner-Mindlin problem. In their work, a discrete minus one norm is used for the least squares method. The main result is that one can choose the subspaces for different variables independently without satisfying the discrete "infsup" condition. Optimal order error estimates are obtained uniformly with respect to the thickness $t$.

An outline of this paper is as follows. After a brief section on preliminaries, we turn in Section 3 to the derivation of error estimates for the two Reissner-Mindlin element families proposed in this paper. The final section contains results of some numerical experiments with these and similar elements. 

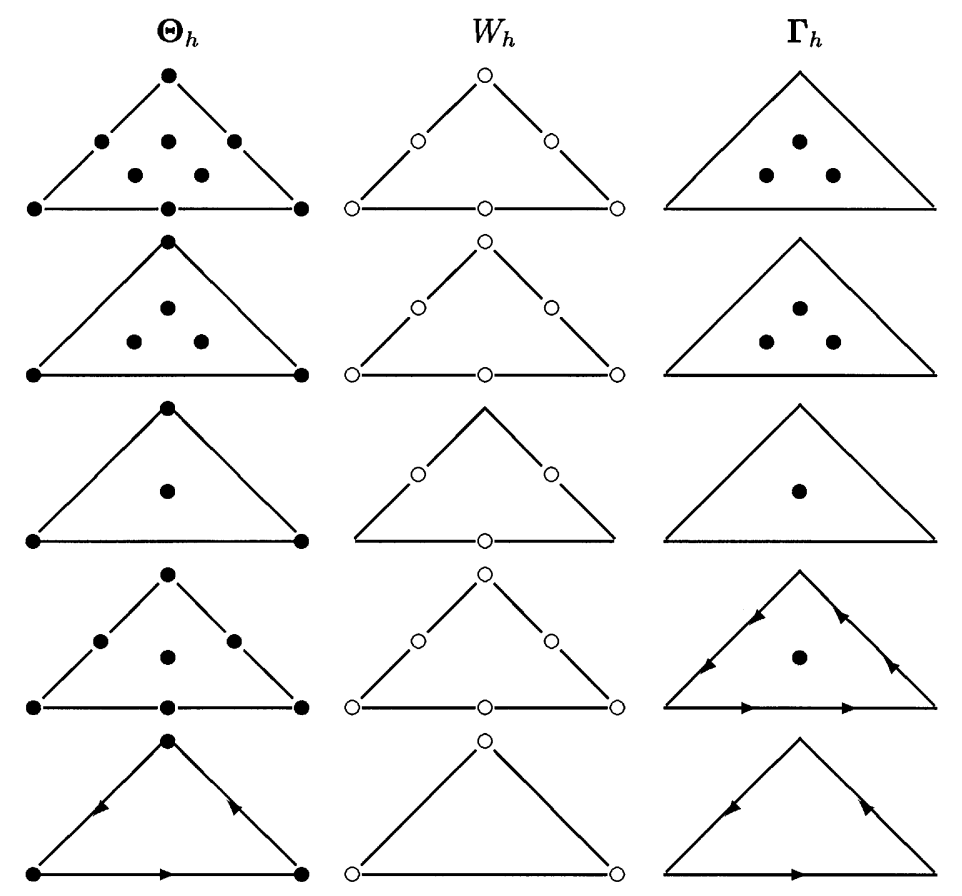

Reference

Zienkiewicz-

Lefebvre [23]

Falk-Tu

Arnold-Falk [2]

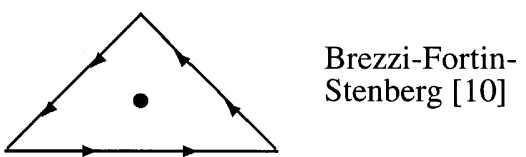

Figure 1. Some finite element schemes for the Reissner-Mindlin plate based on the variational formulation (1.3) and (1.4)

\section{Preliminaries}

We shall assume that $\Omega$ is a convex polygon and that $\left\{\mathcal{T}_{h}\right\}_{\{0<h<1\}}$ is a regular family of triangulations of $\Omega$, where the subscript $h$ refers to the diameter of the largest triangle. Denoting by $\mathcal{P}_{k}(T)$ the set of functions on $T$, which are the restrictions of polynomials of degree less than or equal to $k$, we define the following finite element spaces:

$$
\begin{aligned}
M^{k}: & \left\{\mu \in L^{2}(\Omega):\left.\mu\right|_{T} \in \mathcal{P}_{k}(T) \text { for all } T \in \mathcal{T}_{h}\right\}, \\
M_{0}^{k}: & M^{k} \cap H^{1}(\Omega), \\
\stackrel{\circ}{M}_{0}^{k}: & M^{k} \cap \stackrel{\circ}{H}^{1}(\Omega), \\
B^{k}: & \text { elements of } M_{0}^{k} \text { which vanish on all element edges. }
\end{aligned}
$$

Then, defining for $r=k$ (we shall refer to this as the FT family) or $r=k+1$ (we shall refer to this as the ZL family),

$$
\boldsymbol{\Theta}_{h}=\left[\stackrel{\circ}{M}_{0}^{r} \oplus B^{k+3}\right]^{2}, \quad W_{h}=\stackrel{\circ}{M_{0}^{k+1}}, \quad \boldsymbol{\Gamma}_{h}=\left[M^{k}\right]^{2},
$$

our approximation scheme for either family of elements may be written as follows.

Find $\boldsymbol{\phi}_{h} \in \boldsymbol{\Theta}_{h}, \omega_{h} \in W_{h}, \gamma_{h} \in \boldsymbol{\Gamma}_{h}$ such that

$$
\begin{gathered}
a\left(\boldsymbol{\phi}_{h}, \boldsymbol{\psi}\right)+\left(\boldsymbol{\gamma}_{h}, \operatorname{grad} v-\boldsymbol{\psi}\right)=(g, v) \quad \text { for all } \boldsymbol{\psi} \in \boldsymbol{\Theta}_{h}, v \in W_{h} \\
\left(\operatorname{grad} \omega_{h}-\boldsymbol{\phi}_{h}, \boldsymbol{\eta}\right)-\lambda^{-1} t^{2}\left(\boldsymbol{\gamma}_{h}, \boldsymbol{\eta}\right)=0 \quad \text { for all } \boldsymbol{\eta} \in \boldsymbol{\Gamma}_{h}
\end{gathered}
$$


Introducing the $\boldsymbol{L}^{2}$ projection $\boldsymbol{\Pi}: \boldsymbol{L}^{2} \rightarrow \boldsymbol{\Gamma}_{h}$ and observing that grad $\omega_{h} \in \boldsymbol{\Gamma}_{h}$, we see that

$$
\gamma_{h}=\lambda t^{-2}\left(\operatorname{grad} \omega_{h}-\Pi \phi_{h}\right),
$$

so that $\gamma_{h}$ can be easily eliminated. This leads to the following method in the variables $\phi_{h}$ and $\omega_{h}$.

Find $\phi_{h} \in \boldsymbol{\Theta}_{h}$ and $\omega_{h} \in W_{h}$ such that

$$
a\left(\phi_{h}, \boldsymbol{\psi}\right)+\lambda t^{-2}\left(\operatorname{grad} \omega_{h}-\boldsymbol{\Pi} \phi_{h}, \operatorname{grad} v-\boldsymbol{\Pi} \boldsymbol{\psi}\right)=(g, v) \quad \text { for all } \boldsymbol{\psi} \in \boldsymbol{\Theta}_{h}, v \in W_{h} .
$$

In the above and in the remainder of the paper, we use boldface to denote vector functions, operators, and spaces. To simplify the remainder of the analysis, we shall henceforth set $\lambda=1$.

\section{ERror estimates}

We shall analyze the convergence of the two families of mixed finite elements defined in the previous section by starting with a lemma similar to one developed by R. Durán and E. Liberman in [12].

Lemma 3.1. Let $\omega_{I} \in W_{h}, \phi_{I} \in \mathbf{\Theta}_{h}$, and $\gamma_{I}=t^{-2}\left(\operatorname{grad} \omega_{I}-\Pi \phi_{I}\right)$. Then

$$
\begin{aligned}
& \left\|\phi_{I}-\phi_{h}\right\|_{1}+t\left\|\gamma_{I}-\gamma_{h}\right\|_{0} \\
& \quad \leq C\left(\left\|\phi_{I}-\phi\right\|_{1}+t\left\|\gamma_{I}-\gamma\right\|_{0}+h\|\gamma-\Pi \gamma\|_{0}\right) .
\end{aligned}
$$

Proof. Subtracting (2.1) from (1.1), we get the error equation

$$
\begin{aligned}
& a\left(\phi-\phi_{h}, \boldsymbol{\psi}\right)+\left(\gamma+\gamma_{h}, \operatorname{grad} v-\Pi \boldsymbol{\Pi}\right) \\
& \quad=(\boldsymbol{\gamma}, \boldsymbol{\psi}-\boldsymbol{\Pi} \boldsymbol{\psi}) \text { for all } \boldsymbol{\psi} \in \boldsymbol{\Theta}_{h}, v \in W_{h} .
\end{aligned}
$$

Hence

$$
\begin{aligned}
a\left(\phi_{I}\right. & \left.-\phi_{h}, \psi\right)+\left(\gamma_{I}-\gamma_{h}, \operatorname{grad} v-\Pi \psi\right) \\
& =a\left(\phi_{I}-\phi, \psi\right)+\left(\gamma_{I}-\gamma, \operatorname{grad} v-\Pi \psi\right)+(\gamma, \psi-\Pi \psi) .
\end{aligned}
$$

Taking $\boldsymbol{\psi}-\boldsymbol{\phi}_{I}-\boldsymbol{\phi}_{h} \in \boldsymbol{\Theta}_{h}$ and $v=\omega_{I}-\omega_{h} \in W_{h}$, we have $\operatorname{grad} v-\boldsymbol{\Pi} \boldsymbol{\psi}=$ $t^{2}\left(\gamma_{I}-\gamma_{h}\right)$, and inserting this in (3.2), we get

$$
\begin{aligned}
a\left(\phi_{I}-\right. & \left.\phi_{h}, \phi_{I}-\phi_{h}\right)+t^{2}\left(\gamma_{I}-\gamma_{h}, \gamma_{I}-\gamma_{h}\right) \\
= & a\left(\phi_{I}-\phi, \phi_{I}-\phi_{h}\right)+t^{2}\left(\gamma_{I}-\gamma, \gamma_{I}-\gamma_{h}\right) \\
& +\left(\gamma,\left[\phi_{I}-\phi_{h}\right]=\Pi\left[\phi_{I}-\phi_{h}\right]\right) \\
= & a\left(\phi_{I}-\phi, \phi_{I}-\phi_{h}\right)+t^{2}\left(\gamma_{I}-\gamma, \gamma_{I}-\gamma_{h}\right) \\
& +\left(\gamma-\Pi \gamma,\left[\phi_{I}-\phi_{h}\right]-\Pi\left[\phi_{I}-\phi_{h}\right]\right) .
\end{aligned}
$$

Using the coercivity and continuity of $a(\cdot, \cdot)$, the Schwarz and arithmetic-geometric mean inequalities, and standard approximation properties, we obtain

$$
\begin{aligned}
\| \phi_{I}- & \phi_{h}\left\|_{1}^{2}+t^{2}\right\| \gamma_{I}-\gamma_{h} \|_{0}^{2} \\
\leq & C\left\|\phi_{I}-\phi\right\|_{1}\left\|\phi_{I}-\phi_{h}\right\|_{1}+t^{2}\left\|\gamma_{I}-\gamma\right\|_{0}\left\|\gamma_{I}-\gamma_{h}\right\|_{0} \\
& +\|\gamma-\Pi \gamma\|_{0}\left\|\left(\phi_{I}-\phi_{h}\right)-\Pi\left(\phi_{I}-\phi_{h}\right)\right\|_{0} \\
\leq & C\left(\left\|\phi_{I}-\phi\right\|_{1}^{2}+t^{2}\left\|\gamma_{I}-\gamma\right\|_{0}^{2}+h^{2}\|\gamma-\Pi \gamma\|_{0}^{2}\right) \\
& +\left\|\phi_{I}-\phi_{h}\right\|_{1}^{2} / 2+t^{2}\left\|\gamma_{I}-\gamma_{h}\right\|_{0}^{2} / 2 .
\end{aligned}
$$


Therefore,

$$
\begin{aligned}
& \left\|\phi_{I}-\phi_{h}\right\|_{1}+t\left\|\gamma_{I}-\gamma_{h}\right\|_{0} \\
& \quad \leq C\left(\left\|\phi_{I}-\phi\right\|_{1}+t\left\|\gamma_{I}-\gamma\right\|_{0}+h\|\gamma-\Pi \gamma\|_{0}\right)
\end{aligned}
$$

Next we define some special approximations and examine their properties. Define $\omega_{I}$ to be a standard interpolant of $\omega$ in $W_{h}$ and define $\phi_{I}=\phi_{I}^{0}+\phi_{I}^{b}$, where $\phi_{I}^{0}$ is a standard interpolant of $\phi$ in $\left[M_{0}^{r}\right]^{2}$, and $\phi_{I}^{b} \in\left[B^{k+3}\right]^{2}$ is defined by $\Pi \phi_{I}^{b}=$ $\Pi \phi-\Pi \phi_{I}^{0}-\Pi \operatorname{grad} \omega+\operatorname{grad} \omega_{I}$. Lemma 3.2 shows that $\gamma_{I}=\boldsymbol{\Pi} \gamma$, and Lemma 3.3 shows that $\phi_{I}$ will be a good approximation to $\phi$.

Lemma 3.2. Let $\gamma_{I}$ and $\boldsymbol{\Pi} \boldsymbol{\gamma}$ be as defined above. Then $\boldsymbol{\gamma}_{I}=\boldsymbol{\Pi} \boldsymbol{\gamma}$.

Proof. Using the definitions, we have

$$
\begin{aligned}
\gamma_{I} & =t^{-2}\left(\operatorname{grad} \omega_{I}-\Pi \phi_{I}\right)=t^{-2}\left(\operatorname{grad} \omega_{I}-\Pi \phi_{I}^{0}-\Pi \phi_{I}^{b}\right) \\
& =t^{-2}\left(\operatorname{grad} \omega_{I}-\Pi \phi_{I}^{0}-\Pi \phi+\Pi \phi_{I}^{0}+\Pi \operatorname{grad} \omega-\operatorname{grad} \omega_{I}\right) \\
& =t^{-2} \Pi(\operatorname{grad} \omega-\boldsymbol{\phi})=\Pi \boldsymbol{\gamma} . \quad \square
\end{aligned}
$$

Lemma 3.3. There exists a constant $C$, independent of $h$, such that

$$
\begin{aligned}
& \left\|\phi-\phi_{I}\right\|_{1} \\
& \quad \leq C\left[\left\|\phi-\phi_{I}^{0}\right\|_{1}+h^{-1}\left(\left\|\phi-\phi_{I}^{0}\right\|_{0}+\|\Pi \operatorname{grad} \omega-\operatorname{grad} \omega\|_{0}+\left\|\operatorname{grad}\left(\omega-\omega_{I}\right)\right\|_{0}\right)\right] .
\end{aligned}
$$

In particular, if $k=1$ in the definitions of $\phi_{I}$ and $\omega_{I}$, then

$$
\left\|\phi-\phi_{I}\right\|_{1} \leq C h\left(\|\phi\|_{2}+\|\omega\|_{3}\right) .
$$

Proof. We first prove that

$$
\left\|\phi_{I}^{b}\right\|_{0} \leq C\left\|\boldsymbol{\Pi} \phi_{I}^{b}\right\|_{0} .
$$

Since $\boldsymbol{\phi}_{I}^{b} \in\left[B^{k}\right]^{2}$, we may write it on each triangle $T$ in the form $\lambda_{1} \lambda_{2} \lambda_{3} \boldsymbol{P}_{k}$, where $\boldsymbol{P}_{k}$ is a vector polynomial and $\lambda_{i}$ are the barycentric coordinates of $T$. Since $\lambda_{i} \leq 1$, $i=1,2,3$, we get

$$
\begin{aligned}
\left\|\boldsymbol{\phi}_{I}^{b}\right\|_{0, T}^{2} & =\int_{T} \lambda_{1}^{2} \lambda_{2}^{2} \lambda_{3}^{2} \boldsymbol{P}_{k}^{2} d x d y \leq \int_{T} \lambda_{1} \lambda_{2} \lambda_{3} \boldsymbol{P}_{k}^{2} d x d y \\
& =\left(\boldsymbol{\phi}_{I}^{b}, \boldsymbol{P}_{k}\right)_{T}=\left(\boldsymbol{\Pi} \boldsymbol{\phi}_{I}^{b}, \boldsymbol{P}_{k}\right)_{T} \leq\left\|\boldsymbol{\Pi} \boldsymbol{\phi}_{I}^{b}\right\|_{0, T}\left\|\boldsymbol{P}_{k}\right\|_{0, T} .
\end{aligned}
$$

The result follows from the fact that $\left\|\boldsymbol{\phi}_{I}^{b}\right\|_{0, T}$ and $\left\|\boldsymbol{P}_{k}\right\|_{0, T}$ are equivalent norms on the space of vector polynomials of degree $\leq k$. Hence, we have

$$
\begin{aligned}
\left\|\Pi \phi_{I}^{b}\right\|_{0} & =\left\|\Pi \phi-\Pi \phi_{I}^{0}-\Pi \operatorname{grad} \omega+\operatorname{grad} \omega_{I}\right\|_{0} \\
& \leq\left\|\Pi\left(\phi-\phi_{I}^{0}\right)\right\|_{0}+\|\Pi \operatorname{grad} \omega-\operatorname{grad} \omega\|_{0}+\left\|\operatorname{grad}\left(\omega-\omega_{I}\right)\right\|_{0} \\
& \leq C\left(\left\|\phi-\phi_{I}^{0}\right\|_{0}+\|\Pi \operatorname{grad} \omega-\operatorname{grad} \omega\|_{0}+\left\|\operatorname{grad}\left(\omega-\omega_{I}\right)\right\|_{0}\right) .
\end{aligned}
$$

Now, by the triangle inequality, standard approximation theory, and (3.3), (3.4),

$$
\begin{aligned}
\left\|\phi-\phi_{I}\right\|_{1}= & \left\|\phi-\phi_{I}^{0}-\phi_{I}^{b}\right\|_{1} \leq\left\|\phi-\phi_{I}^{0}\right\|_{1}+\left\|\phi_{I}^{b}\right\|_{1} \\
\leq & \left\|\phi-\phi_{I}^{0}\right\|_{1}+C h^{-1}\left\|\phi_{I}^{b}\right\|_{0} \leq\left\|\phi-\phi_{I}^{0}\right\|_{1}+C h^{-1}\left\|\mathbf{\Pi} \phi_{I}^{b}\right\|_{0} \\
\leq & C\left[\left\|\phi-\phi_{I}^{0}\right\|_{1}\right. \\
& \left.\quad+h^{-1}\left(\left\|\phi-\phi_{I}^{0}\right\|_{0}+\|\Pi \operatorname{grad} \omega-\operatorname{grad} \omega\|_{0}+\left\|\operatorname{grad}\left(\omega-\omega_{I}\right)\right\|_{0}\right)\right] .
\end{aligned}
$$


In particular, if $k=1$ in the definitions of $\phi_{I}$ and $\omega_{I}$, then, using standard approximation theory, we have

$$
\begin{aligned}
\left\|\phi-\phi_{I}\right\|_{1} & \leq C\left[h\|\phi\|_{2}+h^{-1}\left(h^{2}\|\phi\|_{2}+h^{2}\|\operatorname{grad} \omega\|_{2}+h^{2}\|\omega\|_{3}\right)\right] \\
& \leq C h\left(\|\phi\|_{2}+\|\omega\|_{3}\right) . \quad \square
\end{aligned}
$$

Using these results, we now derive our first estimate.

Theorem 3.4. Suppose $(\phi, \omega, \gamma)$ and $\left(\phi_{h}, \omega_{h}, \gamma_{h}\right)$ solve (1.1)-1.2) and (2.1)(2.2), respectively, for some $g \in L^{2}(\Omega)$ and some $t \in(0,1]$. Then there exists a constant $C$, independent of $h$ and $t$, such that

$$
\begin{aligned}
& \left\|\phi-\phi_{h}\right\|_{1}+t\left\|\gamma-\gamma_{h}\right\|_{0} \\
& \leq C\left[\left\|\phi-\phi_{I}^{0}\right\|_{1}+(t+h)\|\gamma-\Pi \gamma\|_{0}\right. \\
& \left.\quad+h^{-1}\left(\left\|\phi-\phi_{I}^{0}\right\|_{0}+\|\Pi \operatorname{grad} \omega-\operatorname{grad} \omega\|_{0}+\left\|\operatorname{grad} \omega-\operatorname{grad} \omega_{I}\right\|_{0}\right)\right] .
\end{aligned}
$$

Proof. The result follows immediately from Lemmas 3.1 3.3, and the triangle inequality.

Applying standard approximation theory, we then obtain

Corollary 3.5. For $2 \leq s \leq k+1$, if $\boldsymbol{\phi} \in \boldsymbol{H}^{s}, \omega \in H^{s+1}$, and $\boldsymbol{\gamma} \in \boldsymbol{H}^{s-1}$, then

$$
\left\|\boldsymbol{\phi}-\phi_{h}\right\|_{1}+t\left\|\boldsymbol{\gamma}-\boldsymbol{\gamma}_{h}\right\|_{0} \leq C h^{s-1}\left(\|\phi\|_{s}+\|\omega\|_{s+1}+\|\boldsymbol{\gamma}\|_{s-2}+t\|\boldsymbol{\gamma}\|_{s-1}\right),
$$

where $C$ is independent of $h$ and $t$.

Note that for $s>5 / 2$, the norms on the right-hand side are not bounded independent of $t$ (cf. [3], [4]). Also note that the estimate for $\phi$ is optimal order for the FT family of elements, but suboptimal by one order for the ZL family.

Remark. If we apply Lemma 3.1 for the ZL family with $\phi_{I}$ and $\omega_{I}$ chosen as standard Lagrange interpolants of $\phi$ and $\omega$, respectively, then, for $2 \leq s \leq k+1$,

$$
\begin{aligned}
\left\|\gamma-\gamma_{I}\right\|_{0} & \leq t^{-2}\left(\left\|\operatorname{grad}\left[\omega-\omega_{I}\right]\right\|_{0}+\|\boldsymbol{\phi}-\boldsymbol{\Pi} \phi\|_{0}+\left\|\boldsymbol{\Pi}\left[\boldsymbol{\phi}-\boldsymbol{\phi}_{I}\right]\right\|_{0}\right) \\
& \leq t^{-2}\left(\left\|\operatorname{grad}\left[\omega-\omega_{I}\right]\right\|_{0}+\|\boldsymbol{\phi}-\boldsymbol{\Pi} \boldsymbol{\phi}\|_{0}+\left\|\boldsymbol{\phi}-\boldsymbol{\phi}_{I}\right\|_{0}\right) \\
& \leq C t^{-2} h^{s}\left(\|\omega\|_{s+1}+\|\boldsymbol{\phi}\|_{s}\right) .
\end{aligned}
$$

Hence, for $2 \leq s \leq k+1$, if $\boldsymbol{\phi} \in \boldsymbol{H}^{s}, \omega \in H^{s+1}$, and $\boldsymbol{\gamma} \in \boldsymbol{H}^{s-1}$,

$$
\begin{aligned}
& \left\|\boldsymbol{\phi}-\boldsymbol{\phi}_{h}\right\|_{1}+t\left\|\boldsymbol{\gamma}-\gamma_{h}\right\|_{0} \\
& \quad \leq C t^{-1} h^{s}\left(\|\boldsymbol{\phi}\|_{s}+t\|\boldsymbol{\phi}\|_{s+1}+\|\omega\|_{s+1}+t\|\gamma\|_{s-1}\right),
\end{aligned}
$$

where $C$ is independent of $h$ and $t$.

This standard estimate improves the order of convergence, but the estimate deteriorates as $t \rightarrow 0$. Combining this result with Corollary 3.5. we get, for the ZL family,

Theorem 3.6. For $2 \leq s \leq k+1$, if $\boldsymbol{\phi} \in \boldsymbol{H}^{s}, \omega \in H^{s+1}$, and $\boldsymbol{\gamma} \in \boldsymbol{H}^{s-1}$, then

$$
\begin{aligned}
& \left\|\phi-\phi_{h}\right\|_{1}+t\left\|\gamma-\gamma_{h}\right\|_{0} \\
& \quad \leq C h^{s-1} \min \left(1, h t^{-1}\right)\left(\|\phi\|_{s}+t\|\phi\|_{s+1}+\|\omega\|_{s+1}+\|\gamma\|_{s-2}+t\|\gamma\|_{s-1}\right) .
\end{aligned}
$$

The above estimate indicates a deterioration of order $h$ from $t=1$ to $t=h$ in the approximation of the derivatives of the rotation, demonstrating locking of order $h^{-1}$. This deterioration is clearly seen in the computational experiments in Section 4 
Theorem 3.7. Under the assumptions of Theorem 3.4, we have

$$
\begin{aligned}
\left\|\phi-\phi_{h}\right\|_{0} \leq & C\left(\|\phi-\Pi \phi\|_{0}+\left(h^{2}+h t\right)\|\gamma-\Pi \gamma\|_{0}+h\left\|\phi-\phi_{I}^{0}\right\|_{1}\right. \\
& \left.+\left\|\phi-\phi_{I}^{0}\right\|_{0}+\|\Pi \operatorname{grad} \omega-\operatorname{grad} \omega\|_{0}+\left\|\operatorname{grad} \omega-\operatorname{grad} \omega_{I}\right\|_{0}\right) .
\end{aligned}
$$

Proof. We introduce the dual problem: Find $\mathbf{\Phi} \in \stackrel{\circ}{\boldsymbol{H}}^{1}(\Omega), W \in \stackrel{\circ}{H}^{1}(\Omega)$, and $\boldsymbol{\zeta} \in$ $\boldsymbol{L}^{2}(\Omega)$ such that

$$
(\boldsymbol{\eta}, \operatorname{grad} W-\boldsymbol{\Phi})-t^{2}(\boldsymbol{\eta}, \boldsymbol{\zeta})=0 \quad \text { for all } \boldsymbol{\eta} \in \boldsymbol{L}^{2}(\Omega) .
$$

Choosing $\boldsymbol{\psi}=\boldsymbol{\phi}-\boldsymbol{\phi}_{h}$ and $v=\omega-\omega_{h}$ in (3.5), we get

$$
\begin{aligned}
\operatorname{grad} v-\boldsymbol{\psi} & =\operatorname{grad} \omega-\operatorname{grad} \omega_{h}-\phi+\phi_{h} \\
& =t^{2} \gamma-t^{2} \gamma_{h}+\phi_{h}-\Pi \phi_{h} .
\end{aligned}
$$

Hence,

$$
\left\|\boldsymbol{\phi}-\boldsymbol{\phi}_{h}\right\|_{0}^{2}=a\left(\boldsymbol{\phi}-\boldsymbol{\phi}_{h}, \boldsymbol{\Phi}\right)+t^{2}\left(\boldsymbol{\gamma}-\boldsymbol{\gamma}_{h}, \boldsymbol{\zeta}\right)+\left(\boldsymbol{\phi}_{h}-\boldsymbol{\Pi} \boldsymbol{\phi}_{h}, \boldsymbol{\zeta}\right) .
$$

Now from (3.1) , for any $(\hat{v}, \hat{\boldsymbol{\psi}}) \in W_{h} \times \boldsymbol{\Theta}_{h}$ and $\hat{\gamma}=t^{-2}(\operatorname{grad} \hat{v}-\boldsymbol{\Pi} \hat{\boldsymbol{\psi}})$ we have

$$
a\left(\phi-\phi_{h}, \hat{\psi}\right)+t^{2}\left(\gamma-\gamma_{h}, \hat{\gamma}\right)-(\gamma, \hat{\psi}-\Pi \hat{\boldsymbol{\psi}}) .
$$

Combining these results and using standard estimates, we get

$$
\begin{aligned}
& \left\|\boldsymbol{\phi}-\boldsymbol{\phi}_{h}\right\|_{0}^{2}=a\left(\boldsymbol{\phi}-\boldsymbol{\phi}_{h}, \boldsymbol{\Phi}-\hat{\boldsymbol{\psi}}\right)+t^{2}\left(\boldsymbol{\gamma}-\boldsymbol{\gamma}_{h}, \boldsymbol{\zeta}-\hat{\boldsymbol{\gamma}}\right) \\
& +\left(\phi_{h}-\Pi \phi_{h}, \zeta\right)+(\gamma, \hat{\psi}-\Pi \hat{\psi}) \\
& =a\left(\phi-\phi_{h}, \boldsymbol{\Phi}-\hat{\boldsymbol{\psi}}\right)+t^{2}\left(\boldsymbol{\gamma}-\gamma_{h}, \boldsymbol{\zeta}-\hat{\gamma}\right) \\
& +\left(\phi_{h}-\phi+\Pi \phi-\Pi \phi_{h}, \boldsymbol{\zeta}\right)+(\phi-\Pi \phi, \zeta) \\
& +(\gamma-\Pi \gamma, \boldsymbol{\Phi}-\Pi \boldsymbol{\Phi})+(\boldsymbol{\gamma}-\boldsymbol{\Pi} \boldsymbol{\gamma}, \hat{\boldsymbol{\psi}}-\boldsymbol{\Phi}-\boldsymbol{\Pi}[\hat{\boldsymbol{\psi}}-\boldsymbol{\Phi}]) \\
& \leq C\left\|\boldsymbol{\phi}-\boldsymbol{\phi}_{h}\right\|_{1}\|\boldsymbol{\Phi}-\hat{\boldsymbol{\psi}}\|_{1}+t^{2}\left\|\boldsymbol{\gamma}-\boldsymbol{\gamma}_{h}\right\|_{0}\|\boldsymbol{\zeta}-\hat{\boldsymbol{\gamma}}\|_{0} \\
& +\left\|\phi_{h}-\phi-\Pi\left(\phi_{h}-\phi\right)\right\|_{0}\|\boldsymbol{\zeta}\|_{0}+\|\phi-\Pi \phi\|_{0}\|\boldsymbol{\zeta}\|_{0} \\
& +\|\boldsymbol{\gamma}-\boldsymbol{\Pi} \boldsymbol{\gamma}\|_{0}\|\mathbf{\Phi}-\boldsymbol{\Pi} \boldsymbol{\Phi}\|_{0}+\|\boldsymbol{\gamma}-\boldsymbol{\Pi} \boldsymbol{\gamma}\|_{0}\|\hat{\boldsymbol{\psi}}-\boldsymbol{\Phi}-\boldsymbol{\Pi}(\hat{\boldsymbol{\psi}}-\mathbf{\Phi})\|_{0} \\
& \leq C\left(\left\|\boldsymbol{\phi}-\boldsymbol{\phi}_{h}\right\|_{1}\|\mathbf{\Phi}-\hat{\boldsymbol{\psi}}\|_{1}+t^{2}\left\|\boldsymbol{\gamma}-\gamma_{h}\right\|_{0}\|\boldsymbol{\zeta}-\hat{\boldsymbol{\gamma}}\|_{0}\right. \\
& +h\left\|\phi_{h}-\phi\right\|_{1}\|\boldsymbol{\zeta}\|_{0}+\|\boldsymbol{\phi}-\boldsymbol{\Pi} \phi\|_{0}\|\boldsymbol{\zeta}\|_{0} \\
& \left.+\|\boldsymbol{\gamma}-\boldsymbol{\Pi} \boldsymbol{\gamma}\|_{0} h^{2}\|\mathbf{\Phi}\|_{2}+\|\boldsymbol{\gamma}-\boldsymbol{\Pi} \boldsymbol{\gamma}\|_{0} h\|\hat{\boldsymbol{\psi}}-\boldsymbol{\Phi}\|_{1}\right) \text {. }
\end{aligned}
$$

By Lemma 3.3 we can choose $\hat{\boldsymbol{\psi}}$ and $\hat{\boldsymbol{\gamma}}$ such that

$$
\|\boldsymbol{\Phi}-\hat{\boldsymbol{\psi}}\|_{1} \leq C h\left(\|\boldsymbol{\Phi}\|_{2}+\|W\|_{3}\right), \quad\|\boldsymbol{\zeta}-\hat{\gamma}\|_{0} \leq C h\|\boldsymbol{\zeta}\|_{1} .
$$

By the regularity proved in the Appendix in 2] we know that

$$
\|W\|_{3}+\|\boldsymbol{\Phi}\|_{2}+\|\boldsymbol{\zeta}\|_{0}+t\|\boldsymbol{\zeta}\|_{1} \leq C\left\|\boldsymbol{\phi}-\boldsymbol{\phi}_{h}\right\|_{0} .
$$


Applying these inequalities and standard approximation theory to the right-hand side of (3.7), we then have

$$
\begin{aligned}
\left\|\boldsymbol{\phi}-\boldsymbol{\phi}_{h}\right\|_{0}^{2} \leq C h\left\|\boldsymbol{\phi}-\boldsymbol{\phi}_{h}\right\|_{0}( & \left\|\boldsymbol{\phi}-\boldsymbol{\phi}_{h}\right\|_{1}+t\left\|\gamma-\gamma_{h}\right\|_{0} \\
& \left.+h^{-1}\|\boldsymbol{\phi}-\Pi \boldsymbol{\phi}\|_{0}+h\|\gamma-\Pi \gamma\|_{0}\right) .
\end{aligned}
$$

The theorem now follows directly from Theorem 3.4 .

Applying standard approximation theory, we then obtain

Corollary 3.8. For $2 \leq s \leq k+1$, if $\boldsymbol{\phi} \in \boldsymbol{H}^{s}, \omega \in H^{s+1}$, and $\boldsymbol{\gamma} \in \boldsymbol{H}^{s-1}$, then

$$
\left\|\phi-\phi_{h}\right\|_{0} \leq C h^{s}\left(\|\phi\|_{s}+\|\omega\|_{s+1}+\|\gamma\|_{s-2}+t\|\gamma\|_{s-1}\right),
$$

where $C$ is independent of $h$ and $t$.

Again note that for $s>5 / 2$, the norms on the right-hand side are not bounded independent of $t$.

Remark. As in the case of the previous error estimate, the estimate for $\phi$ is optimal order for the FT family and can be improved for the ZL family at the expense of introducing a negative power of $t$. To do this, we follow the derivation of (3.7), except that the term $(\boldsymbol{\phi}-\boldsymbol{\Pi} \boldsymbol{\phi}, \boldsymbol{\zeta})$ is estimated by

$$
\begin{aligned}
(\phi-\Pi \phi, \zeta) & =(\phi-\Pi \phi, \zeta-\Pi \zeta) \leq\|\phi-\Pi \phi\|_{0}\|\zeta-\Pi \zeta\|_{0} \\
& \leq C h t^{-1}\|\phi-\Pi \phi\|_{0} t\|\zeta\|_{1} \leq C h t^{-1}\|\phi-\Pi \phi\|_{0}\left\|\phi-\phi_{h}\right\|_{0} .
\end{aligned}
$$

Hence, for $2 \leq s \leq k+1$, if $\boldsymbol{\phi} \in \boldsymbol{H}^{s}, \omega \in H^{s+1}$, and $\gamma \in \boldsymbol{H}^{s-1}$, then

$$
\left\|\phi-\phi_{h}\right\|_{0} \leq C t^{-1} h^{s+1}\left(\|\phi\|_{s}+t\|\phi\|_{s+1}+\|\omega\|_{s+1}+t\|\gamma\|_{s-1}\right),
$$

where $C$ is independent of $h$ and $t$.

Combining this result with Corollary 3.8, we obtain, for the ZL family,

Theorem 3.9. For $2 \leq s \leq k+1$, if $\boldsymbol{\phi} \in \boldsymbol{H}^{s}, \omega \in H^{s+1}$, and $\boldsymbol{\gamma} \in \boldsymbol{H}^{s-1}$, then

$$
\left\|\phi-\phi_{h}\right\|_{0} \leq C h^{s} \min \left(1, h t^{-1}\right)\left(\|\phi\|_{s}+t\|\phi\|_{s+1}+\|\omega\|_{s+1}+\|\gamma\|_{s-2}+t\|\gamma\|_{s-1}\right) \text {. }
$$

We next show that for both families of elements, the derivatives of $\omega$ are approximated to optimal order uniformly in $t$.

Theorem 3.10. Under the assumptions of Theorem 3.4, we have

$$
\begin{aligned}
\left\|\omega-\omega_{h}\right\|_{1} \leq C & \left(\left\|\omega_{I}-\omega\right\|_{1}+\|\boldsymbol{\phi}-\Pi \boldsymbol{\phi}\|_{0}\right. \\
& +\left(h^{2}+h t\right)\|\gamma-\Pi \gamma\|_{0}+h\left\|\phi-\phi_{I}^{0}\right\|_{1} \\
& \left.+\left\|\boldsymbol{\phi}-\boldsymbol{\phi}_{I}^{0}\right\|_{0}+\|\boldsymbol{\Pi} \operatorname{grad} \omega-\operatorname{grad} \omega\|_{0}\right) .
\end{aligned}
$$

Proof. Taking $\boldsymbol{\psi}=0, v=v_{h} \in W_{h}$, and $\boldsymbol{\eta}=\lambda t^{-2} \operatorname{grad} v_{h}$ in (1.1) (1.2) and (2.1)-(2.2), we get

$$
\begin{aligned}
t^{-2}\left(\operatorname{grad} \omega-\boldsymbol{\phi}, \operatorname{grad} v_{h}\right) & =\left(g, v_{h}\right), \\
t^{-2}\left(\operatorname{grad} \omega_{h}-\Pi \phi_{h}, \operatorname{grad} v_{h}\right) & =\left(g, v_{h}\right) .
\end{aligned}
$$

Subtracting (3.9) from (3.8), we deduce that for all $v_{h} \in W_{h}$

$$
\left(\operatorname{grad}\left[\omega-\omega_{h}\right], \operatorname{grad} v_{h}\right)=\left(\phi-\Pi \phi_{h}, \operatorname{grad} v_{h}\right) .
$$


Hence,

$$
\begin{aligned}
& \left(\operatorname{grad}\left[\omega_{I}-\omega_{h}\right], \operatorname{grad} v_{h}\right) \\
& \quad=\left(\operatorname{grad}\left[\omega_{I}-\omega\right], \operatorname{grad} v_{h}\right)+\left(\phi-\Pi \phi_{h}, \operatorname{grad} v_{h}\right) .
\end{aligned}
$$

Choosing $v_{h}=\omega_{I}-\omega_{h}$ in (3.10) and applying the Schwarz inequality, we get

$$
\left\|\operatorname{grad}\left(\omega_{I}-\omega_{h}\right)\right\|_{0} \leq\left\|\operatorname{grad}\left(\omega_{I}-\omega\right)\right\|_{0}+\left\|\phi-\Pi \phi_{h}\right\|_{0} .
$$

It follows easily that

$$
\begin{aligned}
\left\|\omega_{I}-\omega_{h}\right\|_{1} & \leq C\left(\left\|\omega_{I}-\omega\right\|_{1}+\left\|\boldsymbol{\phi}-\boldsymbol{\Pi} \phi_{h}\right\|_{0}\right) \\
& \leq C\left(\left\|\omega_{I}-\omega\right\|_{1}+\|\boldsymbol{\phi}-\boldsymbol{\Pi} \phi\|_{0}+\left\|\boldsymbol{\Pi}\left(\boldsymbol{\phi}-\boldsymbol{\phi}_{h}\right)\right\|_{0}\right) \\
& \leq C\left(\left\|\omega_{I}-\omega\right\|_{1}+\|\boldsymbol{\phi}-\boldsymbol{\Pi} \boldsymbol{\phi}\|_{0}+\left\|\boldsymbol{\phi}-\boldsymbol{\phi}_{h}\right\|_{0}\right) .
\end{aligned}
$$

Applying Theorem 3.7 we obtain

$$
\begin{aligned}
\left\|\omega_{I}-\omega_{h}\right\|_{1} \leq C & \left(\left\|\omega_{I}-\omega\right\|_{1}+\|\boldsymbol{\phi}-\boldsymbol{\Pi} \boldsymbol{\phi}\|_{0}\right. \\
& +\left(h^{2}+h t\right)\|\boldsymbol{\gamma}-\boldsymbol{\Pi} \gamma\|_{0}+h\left\|\boldsymbol{\phi}-\boldsymbol{\phi}_{I}^{0}\right\|_{1} \\
& \left.+\left\|\boldsymbol{\phi}-\boldsymbol{\phi}_{I}^{0}\right\|_{0}+\|\boldsymbol{\Pi} \operatorname{grad} \omega-\operatorname{grad} \omega\|_{0}\right) .
\end{aligned}
$$

The result now follows from the triangle inequality.

Applying standard approximation theory, we then obtain

Corollary 3.11. For $2 \leq s \leq k+1$, if $\boldsymbol{\phi} \in \boldsymbol{H}^{s}, \omega \in H^{s+1}$, and $\boldsymbol{\gamma} \in \boldsymbol{H}^{s-1}$, then

$$
\left\|\omega-\omega_{h}\right\|_{1} \leq C h^{s}\left(\|\phi\|_{s}+\|\omega\|_{s+1}+\|\gamma\|_{s-2}+t\|\gamma\|_{s-1}\right),
$$

where $C$ is independent of $h$ and $t$.

Finally, we derive estimates for the approximation of the shear stress.

Theorem 3.12. Under the assumptions of Theorem 3.4 we have

$$
\begin{array}{r}
\left\|\gamma-\gamma_{h}\right\|_{-1} \leq C\left[\|\gamma-\Pi \gamma\|_{-1}+(h+t)\|\gamma-\Pi \gamma\|_{0}+\left\|\phi-\phi_{I}^{0}\right\|_{1}\right. \\
+h^{-1}\left(\left\|\phi-\phi_{I}^{0}\right\|_{0}+\|\Pi \operatorname{grad} \omega-\operatorname{grad} \omega\|_{0}\right. \\
\left.\left.+\left\|\operatorname{grad}\left(\omega-\omega_{I}\right)\right\|_{0}\right)\right] \\
\left\|\gamma-\gamma_{h}\right\|_{0} \leq C\left[\left(1+t h^{-1}\right)\|\gamma-\Pi \gamma\|_{0}+h^{-1}\left\|\phi-\phi_{I}^{0}\right\|_{1}\right. \\
+h^{-1}\left(\left\|\phi-\phi_{I}^{0}\right\|_{0}+\|\Pi \operatorname{grad} \omega-\operatorname{grad} \omega\|_{0}\right. \\
\left.\left.+\left\|\operatorname{grad}\left(\omega-\omega_{I}\right)\right\|_{0}\right)\right] .
\end{array}
$$

Proof. Choosing $v=0$ in (3.1), we easily see that

$$
\left(\boldsymbol{\Pi} \gamma-\gamma_{h}, \Pi \psi\right)=a\left(\phi-\phi_{h}, \boldsymbol{\psi}\right)-(\boldsymbol{\gamma}, \boldsymbol{\psi}-\boldsymbol{\Pi} \boldsymbol{\psi}) \text { for all } \boldsymbol{\psi} \in \boldsymbol{\Theta}_{h}
$$

For $\boldsymbol{\zeta} \in \stackrel{\circ}{\boldsymbol{H}}^{1}$, let $\boldsymbol{\psi} \in \boldsymbol{\Theta}_{h}$ satisfy

$$
\Pi \boldsymbol{\Pi}=\Pi \boldsymbol{\zeta}, \quad\|\boldsymbol{\zeta}-\boldsymbol{\psi}\|_{0}+h\|\boldsymbol{\psi}\|_{1} \leq C h\|\boldsymbol{\zeta}\|_{1} .
$$

Such a $\psi$ is easily constructed by first using the Clément interpolant and then adding bubble functions to enforce the condition $\Pi \psi=\Pi \zeta$. Then, using (3.11) 
and (3.12), we have

$$
\begin{aligned}
\left(\boldsymbol{\Pi} \boldsymbol{\gamma}-\boldsymbol{\gamma}_{h}, \boldsymbol{\zeta}\right) & =\left(\boldsymbol{\Pi} \boldsymbol{\gamma}-\boldsymbol{\gamma}_{h}, \boldsymbol{\Pi} \boldsymbol{\zeta}\right)=\left(\boldsymbol{\Pi} \boldsymbol{\gamma}-\boldsymbol{\gamma}_{h}, \boldsymbol{\Pi} \boldsymbol{\psi}\right) \\
& =a\left(\boldsymbol{\phi}-\boldsymbol{\phi}_{h}, \boldsymbol{\psi}\right)-(\gamma, \boldsymbol{\psi}-\boldsymbol{\Pi} \boldsymbol{\psi}) \\
& =a\left(\boldsymbol{\phi}-\boldsymbol{\phi}_{h}, \boldsymbol{\psi}\right)-(\boldsymbol{\gamma}-\boldsymbol{\Pi} \boldsymbol{\gamma}, \boldsymbol{\psi}-\boldsymbol{\Pi} \boldsymbol{\psi}) \\
& \leq C\left\|\boldsymbol{\phi}-\boldsymbol{\phi}_{h}\right\|_{1}\|\boldsymbol{\psi}\|_{1}+\|\boldsymbol{\gamma}-\boldsymbol{\Pi} \boldsymbol{\gamma}\|_{0}\|\boldsymbol{\psi}-\boldsymbol{\Pi} \boldsymbol{\psi}\|_{0} \\
& \leq C\|\boldsymbol{\zeta}\|_{1}\left(\left\|\boldsymbol{\phi}-\boldsymbol{\phi}_{h}\right\|_{1}+h\|\boldsymbol{\gamma}-\boldsymbol{\Pi} \boldsymbol{\gamma}\|_{0}\right) .
\end{aligned}
$$

Hence,

$$
\begin{aligned}
\left\|\boldsymbol{\Pi} \boldsymbol{\gamma}-\gamma_{h}\right\|_{-1} & =\sup _{\substack{\boldsymbol{\zeta} \in \boldsymbol{H}^{1}\\
}} \frac{\left(\boldsymbol{\Pi} \boldsymbol{\gamma}-\boldsymbol{\gamma}_{h}, \boldsymbol{\zeta}\right)}{\|\boldsymbol{\zeta}\|_{1}} \\
& \leq C\left(\left\|\boldsymbol{\phi}-\boldsymbol{\phi}_{h}\right\|_{1}+h\|\boldsymbol{\gamma}-\boldsymbol{\Pi} \boldsymbol{\gamma}\|_{0}\right) .
\end{aligned}
$$

The first estimate of the theorem follows directly from the triangle inequality and Theorem 3.4. To obtain the second estimate, let $\boldsymbol{\psi} \in \boldsymbol{\Theta}_{h}$ satisfy

$$
\Pi \boldsymbol{\Pi}=\boldsymbol{\Pi} \boldsymbol{\gamma}-\gamma_{h}, \quad\|\boldsymbol{\psi}\|_{0} \leq C\left\|\boldsymbol{\Pi} \boldsymbol{\gamma}-\gamma_{h}\right\|_{0} .
$$

Such a function is easily constructed using only the bubble functions in $\boldsymbol{\Theta}_{h}$ (cf. (3.3) $)$. Then

$$
\begin{aligned}
\left\|\boldsymbol{\Pi} \boldsymbol{\gamma}-\gamma_{h}\right\|_{0}^{2} & =\left(\boldsymbol{\Pi} \boldsymbol{\gamma}-\gamma_{h}, \boldsymbol{\Pi} \boldsymbol{\psi}\right)=\left(\boldsymbol{\Pi} \boldsymbol{\gamma}-\gamma_{h}, \boldsymbol{\psi}\right) \leq\left\|\boldsymbol{\Pi} \boldsymbol{\gamma}-\boldsymbol{\gamma}_{h}\right\|_{-1}\|\boldsymbol{\psi}\|_{1} \\
& \leq C h^{-1}\left\|\boldsymbol{\Pi} \boldsymbol{\gamma}-\gamma_{h}\right\|_{-1}\|\boldsymbol{\psi}\|_{0} \leq C h^{-1}\left\|\boldsymbol{\Pi} \boldsymbol{\gamma}-\gamma_{h}\right\|_{-1}\left\|\boldsymbol{\Pi} \boldsymbol{\gamma}-\gamma_{h}\right\|_{0}
\end{aligned}
$$

and so, from 3.13),

$$
\begin{aligned}
\left\|\boldsymbol{\Pi} \boldsymbol{\gamma}-\boldsymbol{\gamma}_{h}\right\|_{0} & \leq C h^{-1}\left\|\boldsymbol{\Pi} \boldsymbol{\gamma}-\boldsymbol{\gamma}_{h}\right\|_{-1} \\
& \leq C\left(h^{-1}\left\|\boldsymbol{\phi}-\boldsymbol{\phi}_{h}\right\|_{1}+\|\boldsymbol{\gamma}-\boldsymbol{\Pi} \boldsymbol{\gamma}\|_{0}\right) .
\end{aligned}
$$

The second estimate of the theorem now follows directly from the triangle inequality and Theorem 3.4.

Applying standard approxiamtion theory, we then obtain

Corollary 3.13. For $2 \leq s \leq k+1$, if $\boldsymbol{\phi} \in \boldsymbol{H}^{s}, \omega \in H^{s+1}$, and $\boldsymbol{\gamma} \in \boldsymbol{H}^{s-1}$, then

$$
\begin{aligned}
\left\|\gamma-\gamma_{h}\right\|_{-1} & \leq C h^{s-1}\left(\|\phi\|_{s}+\|\omega\|_{s+1}+\|\gamma\|_{s-2}+t\|\gamma\|_{s-1}\right), \\
\left\|\gamma-\gamma_{h}\right\|_{0} & \leq C h^{s-2}\left(\|\phi\|_{s}+\|\omega\|_{s+1}+\|\gamma\|_{s-2}+t\|\gamma\|_{s-1}\right),
\end{aligned}
$$

where $C$ is independent of $h$ and $t$.

Note that when $k=1$ we obtain a convergence rate of $O(h)$ in the minus one norm, but no positive rate of convergence in $L^{2}$. For $k=2$ and $s=5 / 2$ we get a convergence rate of $O\left(h^{1 / 2}\right)$ in $L^{2}$, where the norms on the solution are all bounded independent of $t$ (cf. [3], [4]). Of course, at the expense of introducing negative powers of $t$, it also follows directly from the definitions, standard estimates, and Corollaries 3.8 and 3.11 that

$$
\begin{aligned}
\left\|\gamma-\gamma_{h}\right\|_{0} & \leq C t^{-2}\left(\left\|\operatorname{grad}\left[\omega-\omega_{h}\right]\right\|_{0}+\|\boldsymbol{\phi}-\mathbf{\Pi} \phi\|_{0}+\left\|\mathbf{\Pi}\left[\boldsymbol{\phi}-\boldsymbol{\phi}_{h}\right]\right\|_{0}\right) \\
& \leq C t^{-2} h^{s}\left(\|\boldsymbol{\phi}\|_{s}+\|\omega\|_{s+1}+\|\gamma\|_{s-2}+t\|\gamma\|_{s-1}\right) .
\end{aligned}
$$

These estimates may be combined to give

$$
\left\|\gamma-\gamma_{h}\right\|_{0} \leq C h^{s-2} \min \left(1, t^{-2} h^{2}\right)\left(\|\phi\|_{s}+\|\omega\|_{s+1}+\|\gamma\|_{s-2}+t\|\gamma\|_{s-1}\right) .
$$




\section{Numerical RESUlts}

In this section we present numerical results for several examples of ReissnerMindlin elements. The aim is to show that the estimates derived in the previous section are sharp, and also to show what happens if the projection into the lower order space is eliminated.

All the examples are done for a unit circular plate which is clamped on its edge and loaded by $g=1$. Because of the symmetry of the plate, we need only discretize one quarter of the domain. The meshes used in our computations are shown in Figures 2 and 3

Essential boundary conditions $\phi_{1}=\phi_{2}=\omega=0$ are applied on the curved portion of the boundary, while on the vertical segment of the boundary $\phi_{1}=0$ is imposed and on the horizontal segment of the boundary $\phi_{2}=0$ is imposed. For the other parameters, we choose Young's modulus $E=1$, the Poisson ratio $\nu=0.3$, the shear correction factor $k=5 / 6$, and the thickness $t=1$ and 0.001 . For each value of the thickness, we compute on a sequence of five meshes with $4 N^{2}$ elements, where $N=1,2,4,8,16$. The exact solution of this problem is given by

$$
\begin{gathered}
\phi_{1}=\frac{x\left(x^{2}+y^{2}-1\right)}{16 D}, \quad \phi_{2}=\frac{y\left(x^{2}+y^{2}-1\right)}{16 D}, \\
\omega=\frac{\left(x^{2}+y^{2}\right)^{2}}{64 D}-\left(x^{2}+y^{2}\right)\left(\frac{\lambda^{-1} t^{2}}{4}+\frac{1}{32 D}\right)+\frac{1}{4} \lambda^{-1} t^{2}+\frac{1}{64 D},
\end{gathered}
$$

where $D=E /\left[12\left(1-\nu^{2}\right)\right]$.
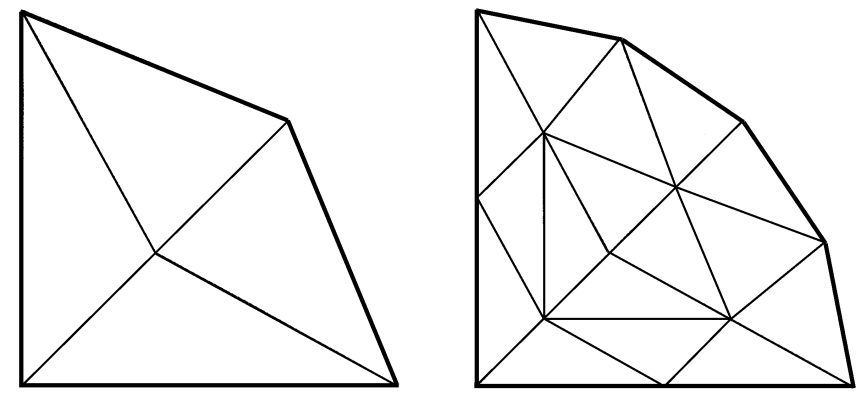

FIgURE 2. Triangular mesh of a quarter circle with 4 elements and 16 elements
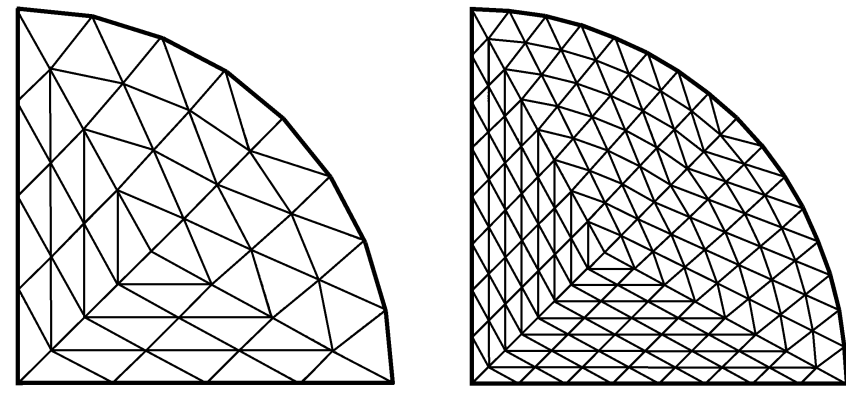

FiguRE 3. Triangular mesh of a quarter circle with 64 elements and 256 elements 
TABLE 1. FT1 for hard clamped plate on a unit circle when $t=1.0$

\begin{tabular}{|crrrrr|}
\multicolumn{7}{c|}{ \% error } \\
\hline component & $N=1$ & $N=2$ & $N=4$ & $N=8$ & $N=16$ \\
\hline$\phi_{1}$ & 58.45 & 16.32 & 4.23 & 1.07 & 0.27 \\
$\phi_{2}$ & 58.45 & 16.32 & 4.23 & 1.07 & 0.27 \\
$\omega$ & 8.02 & 2.30 & 0.60 & 0.15 & 0.04 \\
$\partial \phi_{1} / \partial x$ & 59.88 & 29.40 & 14.56 & 7.26 & 3.63 \\
$\partial \phi_{2} / \partial x$ & 128.73 & 68.58 & 35.25 & 17.79 & 8.92 \\
$\partial \omega / \partial x$ & 9.81 & 2.64 & 0.68 & 0.17 & 0.04 \\
$\partial \phi_{1} / \partial y$ & 128.73 & 68.58 & 35.23 & 17.78 & 8.92 \\
$\partial \phi_{2} / \partial y$ & 59.88 & 29.40 & 14.57 & 7.26 & 3.63 \\
$\partial \omega / \partial y$ & 9.81 & 2.64 & 0.68 & 0.17 & 0.04 \\
\hline
\end{tabular}

We define the FT1 element by choosing $k=1$ in the FT family $(r=1)$, i.e., we choose the usual conforming $\mathcal{P}_{1}$ element augmented by three bubble functions as the space $\boldsymbol{\Theta}_{h}$ to approximate the rotation $\phi$, and $W_{h}$ as the conforming $\mathcal{P}_{2}$ element for the displacement $\omega$. From Corollaries 3.5 3.8, and 3.11, we obtain the following optimal order error estimates for $\phi$ and $\omega$ :

$$
\begin{aligned}
\left\|\phi-\phi_{h}\right\|_{1}+t\left\|\boldsymbol{\gamma}-\gamma_{h}\right\|_{0} & \leq C h\left(\|\phi\|_{2}+\|\omega\|_{3}+t\|\boldsymbol{\gamma}\|_{1}+\|\boldsymbol{\gamma}\|_{0}\right), \\
\left\|\phi-\phi_{h}\right\|_{0}+\left\|\omega-\omega_{h}\right\|_{1} & \leq C h^{2}\left(\|\phi\|_{2}+\|\omega\|_{3}+t\|\boldsymbol{\gamma}\|_{1}+\|\boldsymbol{\gamma}\|_{0}\right) .
\end{aligned}
$$

The numerical results in Tables 1 and 2 show that the method converges for all variables when $t=1.0$ and $t=0.001$. The order of convergence for the first derivative of $\phi_{1}$ and $\phi_{2}$ is 1 , and the order of convergence for the first derivative of $\omega$ and for the $L_{2}$ norm of $\phi$ is 2 , which is consistent with the above estimates. Note that there is no improvement in the order of convergence of $\omega$ itself.

If we choose $k=1$ in the ZL family $(r=2)$, i.e., the usual conforming $\mathcal{P}_{2}$ element augmented by three bubble functions as the space $\boldsymbol{\Theta}_{h}$ to approximate the rotation $\phi$ and $W_{h}$ the conforming $\mathcal{P}_{2}$ element for the displacement $\omega$, we get the triangular plate element ZL1 proposed by Zienkiewicz and Lefebvre [23].

TABLE 2. FT1 for hard clamped plate on a unit circle when $t=0.001$

\begin{tabular}{|crrrrr|}
\multicolumn{7}{c}{ \% error } \\
\hline component & $N=1$ & $N=2$ & $N=4$ & $N=8$ & $N=16$ \\
\hline$\phi_{1}$ & 80.17 & 36.24 & 12.20 & 3.47 & 0.90 \\
$\phi_{2}$ & 80.17 & 36.24 & 12.19 & 3.47 & 0.90 \\
$\omega$ & 78.53 & 36.51 & 12.25 & 3.44 & 0.89 \\
$\partial \phi_{1} / \partial x$ & 79.97 & 54.21 & 32.34 & 17.55 & 8.92 \\
$\partial \phi_{2} / \partial x$ & 146.87 & 99.22 & 57.51 & 29.69 & 14.66 \\
$\partial \omega / \partial x$ & 81.04 & 36.28 & 12.18 & 3.46 & 0.90 \\
$\partial \phi_{1} / \partial y$ & 146.87 & 99.22 & 57.54 & 29.70 & 14.66 \\
$\partial \phi_{2} / \partial y$ & 79.97 & 54.21 & 32.34 & 17.54 & 8.91 \\
$\partial \omega / \partial y$ & 81.04 & 36.28 & 12.17 & 3.46 & 0.90 \\
\hline
\end{tabular}


TABLE 3. ZL1 for hard clamped plate on a unit circle when $t=1.0$

\begin{tabular}{|crrrr|}
\multicolumn{6}{c}{ \% error } \\
\hline component & $N=1$ & $N=2$ & $N=4$ & $N=8$ \\
\hline$\phi_{1}$ & 4.87 & 0.60 & 0.08 & 0.01 \\
$\phi_{2}$ & 4.87 & 0.60 & 0.08 & 0.01 \\
$\omega$ & 1.15 & 0.15 & 0.02 & 0.00 \\
$\partial \phi_{1} / \partial x$ & 11.77 & 2.78 & 0.69 & 0.17 \\
$\partial \phi_{2} / \partial x$ & 26.72 & 6.45 & 1.64 & 0.42 \\
$\partial \omega / \partial x$ & 4.42 & 1.22 & 0.32 & 0.08 \\
$\partial \phi_{1} / \partial y$ & 26.72 & 6.45 & 1.64 & 0.42 \\
$\partial \phi_{2} / \partial y$ & 11.77 & 2.78 & 0.68 & 0.17 \\
$\partial \omega / \partial y$ & 4.42 & 1.22 & 0.32 & 0.08 \\
\hline
\end{tabular}

TABLE 4. ZL1 for hard clamped plate on a unit circle when $t=0.001$

\begin{tabular}{|crrrr|}
\hline \multicolumn{6}{c}{$\%$ error } \\
\hline component & $N=1$ & $N=2$ & $N=4$ & $N=8$ \\
\hline$\phi_{1}$ & 33.94 & 14.16 & 4.01 & 1.03 \\
$\phi_{2}$ & 33.94 & 14.16 & 4.00 & 1.03 \\
$\omega$ & 29.72 & 12.50 & 3.47 & 0.88 \\
$\partial \phi_{1} / \partial x$ & 58.01 & 38.18 & 20.12 & 10.15 \\
$\partial \phi_{2} / \partial x$ & 83.90 & 53.88 & 30.63 & 16.23 \\
$\partial \omega / \partial x$ & 37.45 & 14.61 & 4.07 & 1.04 \\
$\partial \phi_{1} / \partial y$ & 83.90 & 53.88 & 30.68 & 16.25 \\
$\partial \phi_{2} / \partial y$ & 58.01 & 38.18 & 20.11 & 10.14 \\
$\partial \omega / \partial y$ & 37.45 & 14.61 & 4.06 & 1.04 \\
\hline
\end{tabular}

From Theorems 3.6 and 3.9 and Corollary 3.11, we obtain the following error estimates for $\phi, \gamma$, and $\omega$ :

$$
\begin{aligned}
\left\|\phi-\phi_{h}\right\|_{1}+t\left\|\boldsymbol{\gamma}-\gamma_{h}\right\|_{0} & \leq C h \min \left(1, h t^{-1}\right)\left(\|\phi\|_{2}+t\|\phi\|_{3}+\|\omega\|_{3}+t\|\gamma\|_{1}+\|\gamma\|_{0}\right), \\
\left\|\phi-\phi_{h}\right\|_{0} & \leq C h^{2} \min \left(1, h t^{-1}\right)\left(\|\phi\|_{2}+t\|\phi\|_{3}+\|\omega\|_{3}+t\|\gamma\|_{1}+\|\gamma\|_{0}\right), \\
\left\|\omega-\omega_{h}\right\|_{1} & \leq C h^{2}\left(\|\phi\|_{2}+\|\omega\|_{3}+t\|\gamma\|_{1}+\|\gamma\|_{0}\right) .
\end{aligned}
$$

The numerical results in Tables 3 and 4 show that the method converges for all variables when $t=1.0$ and $t=0.001$. Also the order of convergence for the first derivative of $\phi_{1}$ and $\phi_{2}$ is 2 for $t=1$, but deteriorates to 1 when $t=0.001$, while the order of convergence for the first derivative of $\omega$ remains at 2 . There is a similar deterioration in the order of convergence for $\phi$ and $\omega$ from 3 for $t=1$ to 2 when $t=0.001$. This is consistent with the order of convergence estimates given above (the $L^{2}$ estimate for $\omega$ was omitted, since there is no improvement over the $H^{1}$ estimate for $t$ small and the result for $t=1$ is easily obtained by standard methods), and shows that they are sharp. Thus, this element demonstrates locking of order $h^{-1}$ in the approximation of the rotation and its derivatives. 
TABLE 5. P2P2B4 for hard clamped plate on a unit circle when $t=1.0$

\begin{tabular}{|crrrr|}
\hline \multicolumn{7}{c}{$\%$ error } \\
\hline component & $N=1$ & $N=2$ & $N=4$ & $N=8$ \\
\hline$\phi_{1}$ & 5.13 & 0.62 & 0.08 & 0.01 \\
$\phi_{2}$ & 5.13 & 0.62 & 0.08 & 0.01 \\
$\omega$ & 1.21 & 0.15 & 0.02 & 0.00 \\
$\partial \phi_{1} / \partial x$ & 11.95 & 2.79 & 0.69 & 0.17 \\
$\partial \phi_{2} / \partial x$ & 26.46 & 6.43 & 1.64 & 0.42 \\
$\partial \omega / \partial x$ & 4.42 & 1.22 & 0.32 & 0.08 \\
$\partial \phi_{1} / \partial y$ & 26.46 & 46.3 & 1.64 & 0.42 \\
$\partial \phi_{2} / \partial y$ & 11.95 & 2.79 & 0.69 & 0.17 \\
$\partial \omega / \partial y$ & 4.42 & 1.22 & 0.32 & 0.08 \\
\hline
\end{tabular}

TABle 6. P2P2B4 for hard clamped plate on a unit circle when $t=0.001$

\begin{tabular}{|crrrr|}
\multicolumn{6}{c}{$\%$ error } \\
\hline component & $N=1$ & $N=2$ & $N=4$ & $N=8$ \\
\hline$\phi_{1}$ & 89.28 & 98.53 & 91.36 & 44.04 \\
$\phi_{2}$ & 89.28 & 98.53 & 91.35 & 44.01 \\
$\omega$ & 85.04 & 97.57 & 91.04 & 43.51 \\
$\partial \phi_{1} / \partial x$ & 113.01 & 104.75 & 92.76 & 45.61 \\
$\partial \phi_{2} / \partial x$ & 116.96 & 105.07 & 92.81 & 45.75 \\
$\partial \omega / \partial x$ & 89.33 & 98.54 & 91.36 & 44.04 \\
$\partial \phi_{1} / \partial y$ & 116.96 & 105.07 & 92.81 & 46.75 \\
$\partial \phi_{2} / \partial y$ & 113.01 & 104.75 & 92.76 & 45.62 \\
$\partial \omega / \partial y$ & 89.33 & 98.54 & 91.35 & 44.01 \\
\hline
\end{tabular}

TABLE 7. P2P2 for hard clamped plate on a unit circle when $t=1.0$

\begin{tabular}{|crrrr|}
\multicolumn{7}{c}{$\%$ error } \\
\hline component & $N=1$ & $N=2$ & $N=4$ & $N=8$ \\
\hline$\phi_{1}$ & 6.52 & 0.82 & 0.10 & 0.01 \\
$\phi_{2}$ & 6.52 & 0.82 & 0.10 & 0.01 \\
$\omega$ & 1.21 & 0.15 & 0.02 & 0.00 \\
$\partial \phi_{1} / \partial x$ & 14.81 & 3.51 & 0.86 & 0.21 \\
$\partial \phi_{2} / \partial x$ & 23.68 & 5.95 & 1.54 & 0.40 \\
$\partial \omega / \partial x$ & 4.44 & 1.22 & 0.32 & 0.08 \\
$\partial \phi_{1} / \partial y$ & 23.68 & 5.95 & 1.54 & 0.39 \\
$\partial \phi_{2} / \partial y$ & 14.81 & 3.51 & 0.86 & 0.21 \\
$\partial \omega / \partial y$ & 4.44 & 1.22 & 0.32 & 0.80 \\
\hline
\end{tabular}

Finally, Tables 5 and $\left[6\right.$ show the negative effect of dropping the $L^{2}$ projection, and Tables 7 and 8 show the result of experiments with a standard piecewise quadratic approximation for both variables, with no bubbles or projection. 
TABLE 8. P2P2 for hard clamped plate on a unit circle when $t=0.001$

\begin{tabular}{|crrrr|}
\multicolumn{6}{c}{$\%$ error } \\
\hline component & $N=1$ & $N=2$ & $N=4$ & $N=8$ \\
\hline$\phi_{1}$ & 86.80 & 97.06 & 92.21 & 49.38 \\
$\phi_{2}$ & 86.80 & 97.06 & 92.20 & 49.35 \\
$\omega$ & 81.73 & 95.86 & 91.84 & 48.85 \\
$\partial \phi_{1} / \partial x$ & 106.37 & 102.48 & 93.46 & 50.69 \\
$\partial \phi_{2} / \partial x$ & 114.54 & 103.37 & 93.58 & 51.60 \\
$\partial \omega / \partial x$ & 86.91 & 97.07 & 92.21 & 49.38 \\
$\partial \phi_{1} / \partial y$ & 114.54 & 103.37 & 93.58 & 51.60 \\
$\partial \phi_{2} / \partial y$ & 106.37 & 102.48 & 93.47 & 50.71 \\
$\partial \omega / \partial y$ & 86.91 & 97.07 & 92.20 & 49.35 \\
\hline
\end{tabular}

\section{REFERENCES}

1. D. N. Arnold, Innovative finite element methods for plates, Mat. Apl. Comput. 10 (1991), 77-99. MR 93c:65136

2. D. N. Arnold and R. S. Falk, A uniformly accurate finite element method for the ReissnerMindlin plate, SIAM J. Numer. Anal. 26 (1989), 1276-1290. MR 91c:65068]

3. D. N. Arnold and R. S. Falk, The boundary layer for the Reissner-Mindlin plate model, SIAM J. Math. Anal. 21 (1990), 281-312. MR 91c:73053

4. D. N. Arnold and R. S. Falk, Asymptotic analysis of the boundary layer for the ReissnerMindlin plate model, SIAM J. Math. Anal. 27 (1996), 486-514. MR 97i:73064

5. D. N. Arnold and R. S. Falk, Analysis of a linear-linear finite element for the Reissner-Mindlin plate model, Math. Models Methods Appl. Sci. 7 (1997), 217-238. [MR 98b:73034]

6. K. J. Bathe, F. Brezzi, and S. W. Cho, The MITC7 and MITC9 plate bending elements, Comput. \& Structures 32 (1989), 797-841.

7. K. J. Bathe, F. Brezzi and M. Fortin, Mixed-interpolated elements for Reissner-Mindlin plates, Internat. J. Numer. Meth. Engrg. 21 (1989), 1787-1801. MR 90g:73090

8. J. H. Bramble and T. Sun, A negative-norm least squares method for Reissner-Mindlin plates, Math. Comp. 67 (1998), 901-916. MR 99d:73086

9. F Brezzi and M. Fortin, Numerical approximation of Mindlin-Reissner plates, Math. Comp. 47 (1986), 151-158. MR 87g:73057

10. F. Brezzi, M. Fortin, and R. Stenberg, Error analysis of mixed-interpolated elements for Reissner-Mindlin plates, Math. Models Methods Appl. Sci. 1 (1991), 125-151. MR 92e:73030

11. R. Durán, A. Ghioldi, and N. Wolanski, A finite element method for Mindlin-Reissner plate model, SIAM J. Numer. Anal. 28 (1991), 1004-1014. MR 92f:73046

12. R. Durán and E. Liberman, On mixed finite element methods for the Reissner-Mindlin plate model, Math. Comp. 58 (1992), 561-573. MR 92f:65135

13. R. Durán and E. Liberman, On the convergence of a triangular mixed finite element method for Reissner-Mindlin plate, Math. Models Methods Appl. Sci. 6 (1996), 339-352. MR 97e:73064

14. L. Franca and R. Stenberg, A modification of a low-order Reissner-Mindlin plate bending element, The Mathematics of Finite Elements and Applications VII (J. W. Whiteman, ed.), Academic Press, 1991, pp. 425-436. MR 92d:65007

15. L. Franca, R. Stenberg and T. Vihinen, A nonconforming finite element method for the Reissner-Mindlin plate bending model, Proc. 13th IMACS World Conf. Computation and Applied Mathematics, (Vichnevetsky and Miller, eds.), Trinity College, Dublin, 4 (1991), 1907-1908.

16. T. J. R. Hughes and L. Franca, A mixed finite element formulation for Reissner-Mindlin plate theory: Uniform convergence of all higher order spaces, Comp. Methods Appl. Mech. Engrg. 67 (1988), 223-240. MR 89g:73033

17. E. Oñate, F. Zarate and F. Flores, A simple triangular element for thick and thin plate and shell analysis, Internat. J. Numer. Method. Engrg. 37 (1994), 2569-2582. 
18. P. Peisker and D. Braess, Uniform convergence of mixed interpolated elements for ReissnerMindlin plates, RAIRO Modél. Math. Anal. Numér. 26 (1992), 557-574. MR 93j:73070

19. J. Pitkäranta, Analysis of some low-order finite element schemes for Mindlin-Reissner and Kirchoff plates, Numer. Math. 53 (1988), 237-254. MR 89f:65126

20. J. Pitkäranta and M. Suri, Design principles and error analysis for reduced-shear platebending finite elements, Numer. Math. 75 (1996), 223-266. MR 98c:73078

21. R. Stenberg and T. Vihinen, Calculations with some linear elements for Reissner-Mindlin plates, Proc. European Conf. New Advances in Computational Structural Mechanics, Giens, France, (1991), 505-511.

22. T. Tu, Performance of Reissner-Mindlin Elements, Ph.D. Thesis, Dept. Math., Rutgers University, 1998.

23. O. C. Zienkiewicz and D. Lefebvre, A robust triangular plate bending element of the ReissnerMindlin plate, Internat. J. Numer. Methods Engrg. 26 (1998), 1169-1184.

24. O. C. Zienkiewicz, R. L. Taylor, P. Papadopoulos and E. Oñate, Plate bending elements with discrete constraints: New triangular elements, Comput. \& Structures 35 (1990), 505-522.

Department of Mathematics, Rutgers University, Piscataway, New Jersey 08854

E-mail address: falk@math.rutgers.edu

$U R L:$ http://www.math.rutgers.edu/ falk/

Bloomberg Princeton Index Group, 100 Business Park Drive, Skillman, New Jersey 08858

E-mail address: tongtu@bloomberg.net 